\title{
Silica-Grafted Ionic Liquids as Recyclable Catalysts for the Synthesis of 3,4-Dihydropyrano[c]chromenes and Pyrano[2,3-c]pyrazoles
}

\author{
Khodabakhsh Niknam*, Abolhassan Piran \\ Department of Chemistry, Faculty of Sciences, Persian Gulf University, Bushehr, Iran \\ Email: *niknam@pgu.ac.ir, khniknam@gmail.com
}

Received February 10, 2013; revised March 12, 2013; accepted March 20, 2013

Copyright (c) 2013 Khodabakhsh Niknam, Abolhassan Piran. This is an open access article distributed under the Creative Commons Attribution License, which permits unrestricted use, distribution, and reproduction in any medium, provided the original work is properly cited.

\begin{abstract}
Silica-grafted $N$-propyl-imidazolium hydrogen sulfate ([Sipim] $\mathrm{HSO}_{4}$ ) is employed as a recyclable heterogeneous ionic liquid catalyst for the synthesis of 3,4-dihydropyrano[c]-chromenes by the reaction of aromatic aldehydes, malononitrile and 4-hydroxycoumarin at $100^{\circ} \mathrm{C}$ under solvent-free conditions. Also, heterogeneous ionic liquid catalyst was used for the synthesis of pyrano[2,3-c]-pyrazoles by the reaction of aromatic aldehydes, malononitrile and 3-methyl-l-phenyl5-pyrazolone at $110^{\circ} \mathrm{C}$ under solvent-free conditions. The heterogeneous ionic liquid showed much the same efficiency when used in consecutive reaction runs.
\end{abstract}

Keywords: Silica-Grafted N-Propyl-Imidazolium Hydrogen Sulfate; Aldehydes; Pyrano[2,3-c]-Pyrazoles; 3,4-Dihydropyrano[c]-Chromenes; Solvent-Free, Heterogeneous Ionic Liquid Catalysts

\section{Introduction}

In the recent years, ionic liquids were used as solvents due to their particular properties, such as the ability to dissolve many organic and inorganic substances and undetectable vapor pressure [1]. In addition, Brønsted acidic task-specific ionic liquids (BAILs), such as those possessing $\mathrm{HSO}_{4}^{-}$as a counter anion find a broad application in organic synthesis, acting as both solvents and catalysts. Keim and co-workers reported the synthesis of 1-butyl-3-methylimidazolium hydrogen sulfate ([bmim] $\mathrm{HSO}_{4}$ ) [2]. In addition, in the year of 2002 ([bmim] $\mathrm{HSO}_{4}$ ) was used as a catalyst in the Friedel-Crafts alkylation [3]. The other applications of these acidic ionic liquids such as acetalization and thioacetalization of carbonyl compounds [4], Fischer indole synthesis [5], acetylation of alcohols and phenols [6], preparation of azides from alcohols [7], selective nitration of phenols [8], synthesis of 1,8-dioxo-octahydroxanthenes [9], formylation of alcohols [10], synthesis of polysubstituted quinolines [11], have been proceeded with very good yields and selectivities. Recently, immobilization of acidic ionic liquids on solid supports has been designed and it can offer important advantages in handling, separation and reuse proce-

*Corresponding author. dures. Based on economic criteria, it is desirable to minimize the amount of ionic liquid utilized in a potential process. Immobilized acidic ionic liquids have been used as novel solid catalysts, e.g., for esterification, nitration reactions [12], acetal formation [13], Baeyer-Villiger reaction [14], synthesis of $\alpha$-aminonitriles [15] and bispyrazolones [16].

Dihydropyrano[c]chromenes and their derivatives are of considerable interest as they possess a wide range of biological properties [17], such as spasmolytic, diuretic, anti-coagulant, anti-cancer, and anti-anaphylactic activity $[18,19]$. In addition, they can be used as cognitive enhancers, for the treatment of neurodegenerative diseases, including Alzheimer's disease, Parkinson's disease, AIDS associated dementia and Down's syndrome as well as for the treatment of schizophrenia and myoclonus [20]. Also, a number of 2-amino-4H-pyranes are useful as photoactive materials [21]. A number of methods have been reported for the synthesis of 3,4-dihydropyrano[c] chromenes such as piperidine in organic solvent, i.e. ethanol and pyridine [22], $\mathrm{K}_{2} \mathrm{CO}_{3}$ under microwave irradiation [23], diammonium hydrogen phosphate [24], tetrabutylammonium bromide [25], sodium dodecyl sulfate [26], DBU [27], morpholine [28], $\alpha-\mathrm{Fe}_{2} \mathrm{O}_{3}$ nanopatricles [29], $\mathrm{CuO}$ nanopatricles [30], and silica-bonded $N$-propyl- 
piperazine sodium propionate [31].

In continuation of our studies on the design and application of acidic ionic liquids or silica-grafted ionic liquids as catalyst in organic transformations (Scheme 1) [8-11,15,16], herein, we describe the application of silicagrafted $N$-propyl-imidazolium hydrogen sulfate ([Sipim] $\mathrm{HSO}_{4}$ ) in the synthesis of pyrano[c]chromenes and pyrano[3,4c]pyrazoles.

\section{Results and Discussion}

\subsection{Synthesis of Dihydropyrano[c]chromenes}

To study the effect of catalyst loading on the synthesis of 2-amino-4-aryl-5-oxo-4H,5H-pyrano[3,2-c]chromene-3carbonitrile the condensation reaction of malononitrile, 4-chlorobenzaldehyde, and 4-hydroxycoumarin was chosen as a model reaction (Table 1). The results show clearly that ionic liquids and silica-grafted ionic liquids (SGILs) are effective catalysts for this condensation and the optimal amount of SGILs was $0.1 \mathrm{~g}$ per $1 \mathrm{mmol}$ of aldehyde under solvent-free conditions at $100^{\circ} \mathrm{C}$. The best result was obtained in the presence of $[\mathrm{Sipim}] \mathrm{HSO}_{4}$. This condensation was carried out with the lower amounts of [Sipim] $\mathrm{HSO}_{4} 0.05 \mathrm{~g}$ and $0.07 \mathrm{~g}$ and the corresponding product was obtained in $88 \%$ and $91 \%$ yield (Table 1, entries 4 and 5).

Also, this three-component condensation was accomplished in the presence of $[\mathrm{Sipim}] \mathrm{HSO}_{4}$ in ethanol and water at reflux conditions in longer reaction time and lower yield (Table 1, entries 1 and 2). Moreover, the model reaction was examined under solvent-free conditions at $80^{\circ} \mathrm{C}$ gave $\mathbf{1 b}$ after $90 \mathrm{~min}$ in $80 \%$ yield (Table $\mathbf{1}$, entry 8$)$. The model reaction was reacted in the presence of $N$-(3-silicapropyl) imidazolium chloride ([Sipim]Cl), $N$-(3-silicapropyl) imidazolium dihydrogen phosphate ([Sipim] $\mathrm{H}_{2} \mathrm{PO}_{4}$ ), and $N$-(3-silicapropyl) imidazolium triflate ([Sipim]OTf), under solvent-free conditions at $100^{\circ} \mathrm{C}$ in $80 \%, 80 \%$, and $75 \%$ yield respectively (Table 1, entries 10-12). In addition, the same model reaction was carried out in the presence of ionic liquids such as imidazolium chloride, imidazolium hydrogen sulfate, methylimidazolium hydrogen sulfate, and imidazolium bromide under solvent-free and at $100^{\circ} \mathrm{C}$ in $85 \%, 80 \%, 80 \%$, and $85 \%$ yield respectively (Table 1, entries 13-17).

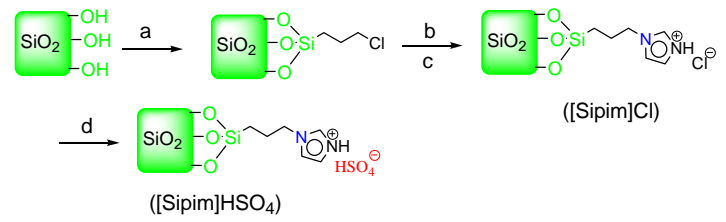

a) $(\mathrm{MeO})_{3} \mathrm{Si}-\left(\mathrm{CH}_{2}\right)_{3}-\mathrm{Cl}$, toluene(dry), $\mathrm{Et}_{3} \mathrm{~N}$, reflux, $48 \mathrm{~h}$; b) Imidazole, toluene (dry), $24 \mathrm{~h}$, reflux c) Dry under vaccum $\left(50^{\circ} \mathrm{C}\right), 4 \mathrm{~h}$; d) dry $\mathrm{CH}_{2} \mathrm{Cl}_{2}, \mathrm{H}_{2} \mathrm{SO}_{4}(97 \%), 48 \mathrm{~h}$, reflux

Scheme 1. Preparation of silica-grafted propyl imidazolium hydrogen sulfate ([Sipim] $\left.\mathrm{HSO}_{4}\right)$.
Table 1. Investigation the effect of catalyst and solvent on the reaction of 4-chlorobenzaldehyde, malononitrile and 4hydroxycoumarin.

\begin{tabular}{|c|c|c|c|c|c|}
\hline Entry & Catalyst & $\begin{array}{l}\text { Catalyst } \\
\text { loading (g) }\end{array}$ & $\begin{array}{c}\text { Solvent/ Temp. } \\
\left({ }^{\circ} \mathrm{C}\right)\end{array}$ & $\begin{array}{l}\text { Time } \\
(\mathrm{min})\end{array}$ & $\begin{array}{l}\text { Yield } \\
(\%)^{\mathrm{a}}\end{array}$ \\
\hline 1 & 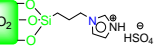 & 0.1 & EtOH/Reflux & 75 & 75 \\
\hline 2 & 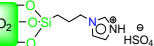 & 0.1 & $\mathrm{H}_{2} \mathrm{O} /$ Reflux & 75 & 75 \\
\hline 3 & NH & 0.1 & $\begin{array}{l}\text { EtOH/ } / \mathrm{H}_{2} \mathrm{O} \\
(1: 1) / \text { Reflux }\end{array}$ & 60 & 86 \\
\hline 4 & 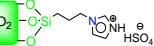 & 0.05 & Solvent-free/100 & 120 & 88 \\
\hline 5 & 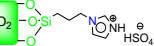 & 0.07 & Solvent-free/100 & 90 & 91 \\
\hline 6 & 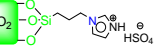 & 0.1 & Solvent-free/100 & 30 & 95 \\
\hline 7 & 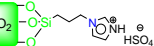 & 0.15 & Solvent-free/100 & 30 & 95 \\
\hline 8 & 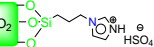 & 0.1 & Solvent-free/80 & 90 & 80 \\
\hline 9 & 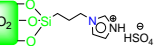 & 0.1 & Solvent-free/120 & 30 & 95 \\
\hline 10 & Nons & 0.1 & Solvent-free/100 & 30 & 80 \\
\hline 11 & 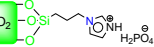 & 0.1 & Solvent-free/100 & 30 & 80 \\
\hline 12 & 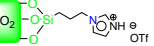 & 0.1 & Solvent-free/100 & 60 & 75 \\
\hline 13 & $\hat{D}_{\mathrm{NH}}^{\oplus} \stackrel{\ominus}{\mathrm{Cl}}$ & 0.2 & Solvent-free/100 & 120 & 85 \\
\hline 14 & $\stackrel{\mathrm{HN}}{\mathrm{e}} \mathrm{\oplus}^{\mathrm{N}} \mathrm{H} \mathrm{HSO}_{4}$ & 0.2 & Solvent-free/100 & 150 & 80 \\
\hline 15 & $\mathrm{H}_{3} \mathrm{CN} \widehat{\mathrm{C}} \hat{\mathrm{N}}^{\oplus \mathrm{NH}} \mathrm{HSO}_{4}$ & 0.2 & Solvent-free/100 & 210 & 80 \\
\hline 16 & 䎡 & 0.2 & Solvent-free/100 & 120 & 75 \\
\hline 17 & HN $\widehat{0}{ }^{\oplus} \mathrm{NH}$ & 0.2 & Solvent-free/100 & 120 & 85 \\
\hline
\end{tabular}

${ }^{\mathrm{a}}$ Isolated Yield.

The synthesis of 2-amino-4-aryl-5-oxo-4H,5H-pyrano[3,2-c]chromene-3-carbonitrile was achieved by the threecomponent condensation of an aromatic aldehyde, malononitrile, and 4-hydroxycoumarin in the presence of [Sipim] $\mathrm{HSO}_{4}\left(0.1 \mathrm{~g}, 0.08 \mathrm{mmol}\right.$ of $\mathrm{H}^{+}$[15]) under solventfree conditions at $100^{\circ} \mathrm{C}$ (Table 2).

Thereafter, a series of different 3,4-dihydropyrano[c]chromene derivatives were prepared successfully from different aromatic aldehydes bearing electron-withdrawing and electron donating groups, 4-hydroxycoumarin and malononitrile under solvent-free conditions. Electron-withdrawing groups such as 3-nitro, 4-nitro, and 2-nitro-benzaldehyde reacted under optimized conditions into corresponding $\mathbf{1 f}, \mathbf{1 g}$, and $\mathbf{1 h}$ in $93 \%$, 90\%, and 89\% yield after $30 \mathrm{~min}$ (Table 2, entries 6-8). Electrondo- 
Table 2. [Sipim] $\mathrm{HSO}_{4}$ catalyzed synthesis of dihydropyrano [c]chromene derivatives. ${ }^{a}$

\begin{tabular}{|c|c|c|c|}
\hline 2 & 3 & & 1 \\
\hline Entry & $\mathrm{Ar}$ & Product & Yield (\%) ${ }^{\mathrm{b}}$ \\
\hline 1 & $\mathrm{C}_{6} \mathrm{H}_{5^{-}}$ & $1 \mathrm{a}$ & 94 \\
\hline 2 & $4-\mathrm{ClC}_{6} \mathrm{H}_{4}-$ & $1 b$ & $95,95,93,91,90^{c}$ \\
\hline 3 & $3-\mathrm{ClC}_{6} \mathrm{H}_{4}-$ & 1c & 93 \\
\hline 4 & $4-\mathrm{BrC}_{6} \mathrm{H}_{4}^{-}$ & $1 \mathrm{~d}$ & 94 \\
\hline 5 & $2,4-(\mathrm{Cl})_{2} \mathrm{C}_{6} \mathrm{H}_{3^{-}}$ & $1 \mathrm{e}$ & 90 \\
\hline 6 & $3-\mathrm{O}_{2} \mathrm{NC}_{6} \mathrm{H}_{4}^{-}$ & 1f & 93 \\
\hline 7 & $4-\mathrm{O}_{2} \mathrm{NC}_{6} \mathrm{H}_{4}^{-}$ & $1 \mathrm{~g}$ & 90 \\
\hline 8 & $2-\mathrm{O}_{2} \mathrm{NC}_{6} \mathrm{H}_{4}^{-}$ & $1 \mathrm{~h}$ & 89 \\
\hline 9 & $4-\mathrm{MeC}_{6} \mathrm{H}_{4^{-}}$ & $1 \mathrm{i}$ & 94 \\
\hline 10 & $3,4,5-\left(\mathrm{CH}_{3} \mathrm{O}\right)_{3} \mathrm{C}_{6} \mathrm{H}_{2}$ & $1 \mathrm{j}$ & 89 \\
\hline 11 & 4-HO- $\mathrm{C}_{6} \mathrm{H}_{4}^{-}$ & $1 \mathrm{k}$ & 93 \\
\hline
\end{tabular}

${ }^{a}$ Reaction conditions: 4-hydroxycoumarin ( $\left.1 \mathrm{mmol}\right)$, malononitrile $(1 \mathrm{mmol})$, aldehyde $(1 \mathrm{mmol})$, solvent-free conditions at $100^{\circ} \mathrm{C}$ for $30 \mathrm{~min}$. ${ }^{\mathrm{b}}$ Isolated yield. ${ }^{\mathrm{C}}$ The recovered [Sipim] $\mathrm{HSO}_{4}$ was used as catalyst.

nating groups such as 4-Me, 3,4,5-(MeO) $)_{3}$-benzaldehyde were treated with malononitrile and 4-hy- droxycoumarin under optimized conditions gave corresponding products $\mathbf{1 i}$ and $\mathbf{1 j}$ in high yields (Table 2, entries 9 and 10). The results clearly indicate that reactions can tolerate a wide range of differently substituted aromatic aldehydes.

The possibility of recycling the catalyst was examined using the reaction of malononitrle, 4-chlorobenzaldehyde and 4-hydroxycoumarin under the optimized conditions. Upon completion, the reaction mixture was washed with warm ethanol $(3 \times 30 \mathrm{~mL})$. The recovered catalyst was dried and reused for subsequent runs. The recycled catalyst could be reused fourth times without any additional treatment. No observation of any appreciable loss in the catalytic activity of [Sipim] $\mathrm{HSO}_{4}$ was made (Table 2, entry 2).

\subsection{Synthesis of Pyrano[2,3-c]pyrazoles}

Condensed pyrazoles are also biologically interesting compounds and their chemistry has recently received considerable attention [32,33]. Several pyrano[2,3-c] pyrazoles are reported to have useful biological effects, such as analgesic and anti-inflammatory activities [34]. Moreover, the biological activity of fused azoles has led to intensive research on their synthesis [35,36]. Recently, three component one-pot condensation of 3-methyl-1-
phenyl-1H-pyrazol-5(4H)-one, aldehydes and malononitrile for the construction of 1,4-dihydropy-rano[2,3-c] pyrazole derivatives has been reported under different conditions [37-41]. However, most of the reported methods have their drawbacks. For example, hexadecyltrimethylammonium bromide (HTMAB) is a harmful and irritant catalyst which is dangerous for the environment [39]. In addition, the reusability of the catalysts such as D,L-proline is not reported in a number of cases [40]. Heteropoly acids, silica-supported bases or acids have been reported the synthesis of 1,4-dihydropyrano[2,3-c] pyrazole derivatives via multi-component condensation reaction [42-48].

The scope of this methodology was extended to the synthesis of pyrano[2,3-c]pyrazols. Initially three-component condensation reaction of malononitrile, 4-chlorobenzaldehyde, and 3-methyl-1-phenyl-1H-pyrazol-5 (4H)one was chosen as a model reaction and the effect of different catalysts was investigated at $110^{\circ} \mathrm{C}$ under solvent-free conditions (Table 3).

Again, the results show clearly that ionic liquids and silica-grafted ionic liquids (SGILs) are effective catalysts

Table 3. Investigation the effect of catalyst on the reaction of 4-chlorobenzaldehyde, malononitrile and 3-methyl-1phenyl-1H-pyrazol-5(4H)-one at $110^{\circ} \mathrm{C}$ under solvent-free conditions.

\begin{tabular}{|c|c|c|c|c|}
\hline Entry & Catalyst & $\begin{array}{c}\text { Catalyst } \\
\text { loading (g) }\end{array}$ & Time (min) & Yield (\%) \\
\hline 1 & - & 0.05 & 120 & 80 \\
\hline 2 & م & 0.07 & 120 & 85 \\
\hline 3 & 然 & 0.1 & 90 & 90 \\
\hline 4 & 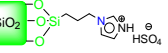 & 0.15 & 90 & 95 \\
\hline 5 & 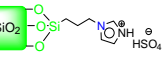 & 0.2 & 90 & 95 \\
\hline 6 & 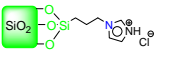 & 0.15 & 100 & 90 \\
\hline 7 & 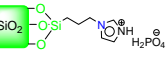 & 0.15 & 100 & 90 \\
\hline 8 & 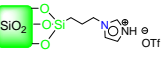 & 0.15 & 120 & 70 \\
\hline 13 & [0 & 0.2 & 120 & 85 \\
\hline 14 & $\stackrel{\ominus}{\mathrm{HSO}_{4}}$ & 0.2 & 150 & 80 \\
\hline 15 & $\mathrm{H}_{3} \mathrm{CN} \widehat{\mathrm{C}} \widehat{\mathrm{C}}^{\mathrm{NH}} \mathrm{HSO}_{4}$ & 0.2 & 150 & 80 \\
\hline 16 & $\mathrm{HN} \widehat{0} \mathrm{NHH}_{2} \mathrm{PO}_{4}$ & 0.2 & 150 & 70 \\
\hline 17 & HN & 0.2 & 120 & 85 \\
\hline
\end{tabular}

${ }^{\mathrm{a}}$ Isolated Yield. 
for this condensation and the optimal amount of SGILs was $0.15 \mathrm{~g}$ per $1 \mathrm{mmol}$ of aldehyde under solvent-free conditions at $110^{\circ} \mathrm{C}$.

A range of different substituted groups on aromatic aldehydes involving electron-withdrawing groups such as 3-nitro, 4-nitro, and electron-donating groups such as 3,4dimethoxy-benzaldehyde reacted under optimized conditions into corresponding $\mathbf{5 f}, \mathbf{5 g}$, and $\mathbf{5 h}$ in $90 \%$, 88\%, and 90\% yield after 90 min (Table 4, entries 6-8). 4-Hydroxy-benzaldehyde was treated with malononitrile and 3-methyl-1-phenyl-1H-pyrazol-5(4H)-one under optimized conditions gave corresponding products $5 \mathbf{i}$ in $90 \%$ yield (Table 4, entry 9).

The possibility of recycling the catalyst was examined using the reaction of malononitrle, 4-chlorobenzaldehyde and 3-methyl-1-phenyl-1H-pyrazol-5(4H)-one under the optimized conditions. Upon completion, the reaction mixture was washed with warm ethanol $(3 \times 30 \mathrm{~mL})$. The recovered catalyst was dried and reused for subsequent runs. The recycled catalyst could be reused fourth times without any additional treatment. No observation of any appreciable loss in the catalytic activity of $[\mathrm{Sipim}] \mathrm{HSO}_{4}$ was made (Table 4, entry 2).

\section{Experimental Section}

\subsection{General}

Chemicals were purchased from Fluka, Merck and Aldrich Chemical Companies. All the products were char-

Table 4. [Sipim] $\mathrm{HSO}_{4}$ catalyzed synthesis of dihydropyrano [2,3-c]pyrazole derivatives. ${ }^{\mathrm{a}}$
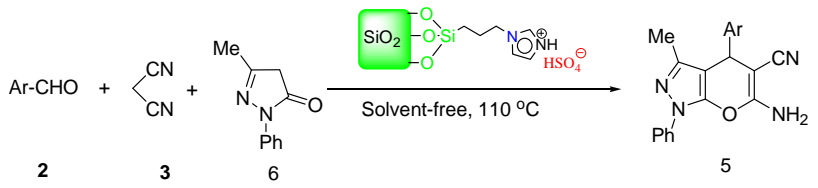

\begin{tabular}{cccc}
\hline Entry & $\mathrm{Ar}$ & Product & ${\text { Yield }(\%)^{\mathrm{b}}}^{2}$ \\
\hline 1 & $\mathrm{C}_{6} \mathrm{H}_{5^{-}}$ & $5 \mathrm{a}$ & 92 \\
3 & $4-\mathrm{ClC}_{6} \mathrm{H}_{4^{-}}$ & $5 \mathrm{~b}$ & $95,94,92,90,89^{\mathrm{c}}$ \\
4 & $2,4-(\mathrm{Cl})_{2} \mathrm{C}_{6} \mathrm{H}_{3^{-}}$ & $5 \mathrm{c}$ & 89 \\
5 & $4-\mathrm{BrC}_{6} \mathrm{H}_{4^{-}}$ & $5 \mathrm{~d}$ & 95 \\
6 & $4-\mathrm{FC}_{6} \mathrm{H}_{4^{-}}$ & $5 \mathrm{e}$ & 90 \\
7 & $3-\mathrm{O}_{2} \mathrm{NC}_{6} \mathrm{H}_{4^{-}}$ & $5 \mathrm{f}$ & 90 \\
8 & $4-\mathrm{O}_{2} \mathrm{NC}_{6} \mathrm{H}_{4^{-}}$ & $5 \mathrm{~g}$ & 88 \\
9 & $3,4-\left(\mathrm{CH}_{3} \mathrm{O}_{2} \mathrm{C}_{6} \mathrm{H}_{3-}\right.$ & $5 \mathrm{~h}$ & 90 \\
\hline
\end{tabular}

${ }^{a}$ Reaction conditions: 3-methyl-1-phenyl-1H-pyrazol-5(4H)-one (1 mmol), malononitrile ( $1 \mathrm{mmol})$, aldehyde $(1 \mathrm{mmol})$, solvent-free conditions at 110 ${ }^{\circ} \mathrm{C}$ for 90 min. ${ }^{b}$ Isolated yield. ${ }^{\mathrm{C}}$ The recovered [Sipim] $\mathrm{HSO}_{4}$ was used as catalyst. pyl-imidazolium hydrogen sulfate ([Sipim] $\left.\mathrm{HSO}_{4}\right)$ was acterized by comparison of their IR, ${ }^{1} \mathrm{H}$ NMR and ${ }^{13} \mathrm{C}$ NMR spectroscopic data and their melting points with the reported values [24-31,38-41]. Silica-grafted $N$-proprepared according to our previous reported procedure [15].

\subsection{General Procedure for the Synthesis of 3,4-Dihydropyrano[c]chromenes}

To a mixture of aromatic aldehyde ( $1 \mathrm{mmol})$, malonitrile (1 mmol), and 4-hydroxycoumarin (1 mmol), catalyst [Sipim] $\mathrm{HSO}_{4}\left(0.1 \mathrm{~g}, 0.08 \mathrm{mmol}\right.$ of $\left.\mathrm{H}^{+}\right)$was added and the mixture was heated at $100^{\circ} \mathrm{C}$ under solvent-free conditions. After completion of the reaction, as indicated by TLC, ethanol $(10 \mathrm{~mL})$ was added and the reaction mixture was filtered. The remaining was washed with warm ethanol $(3 \times 5 \mathrm{~mL})$ in order to separate heterogeneous catalyst. After cooling the crude products were precipitated. The crude products were purified by recrystallization from ethanol (95\%). The recovered catalyst was dried and reused for subsequent runs.

2-Amino-4-phenyl-4,5-dihydro-5-oxopyrano[3,2-c]c hromene-3-carbonitrile 1a: $\mathrm{mp} 258^{\circ} \mathrm{C}-260^{\circ} \mathrm{C}$, (Lit.: $256^{\circ} \mathrm{C}-258^{\circ} \mathrm{C}$, [24]). ${ }^{1} \mathrm{H}$ NMR (400 MHz, DMSO-d $\left.{ }_{6}\right): \delta$ (ppm) 4.46 (s, $1 \mathrm{H}), 7.23$ - 7.27 (m, $3 \mathrm{H}), 7.31$ - 7.35 (m, $2 \mathrm{H}), 7.43-7.52(\mathrm{~m}, 2 \mathrm{H}), 7.72\left(\mathrm{dt}, 1 \mathrm{H}, J_{1}=7.8 \mathrm{~Hz}, J_{2}=\right.$ $1.6 \mathrm{~Hz}$ ), $7.92\left(\mathrm{dd}, 1 \mathrm{H}, J_{1}=8.0 \mathrm{~Hz}, J_{2}=0.8 \mathrm{~Hz}\right) .{ }^{13} \mathrm{C}$ NMR (100 MHz, DMSO-d $\left.{ }_{6}\right): \delta$ (ppm) 58.41, 104.47, 113.42, 117.06, 119.68, 122.98, 125.18, 127.62, 128.10, $129.01,133.45,143.80,152.60,153.89,158.36,158.40$, 160.05 .

2-Amino-4-(4-chlorophenyl)-4,5-dihydro-5-oxopyra no[3,2-c]chromene-3-carbonitrile 1b: mp $263^{\circ} \mathrm{C}$ $265^{\circ} \mathrm{C}$, (Lit.: $252^{\circ} \mathrm{C}-255^{\circ} \mathrm{C}$, [28]). ${ }^{1} \mathrm{H}$ NMR (400 MHz, DMSO-d $\left.{ }_{6}\right): \delta(\mathrm{ppm}) 4.49(\mathrm{~s}, 1 \mathrm{H}), 7.31(\mathrm{~d}, 2 \mathrm{H}, J=8.4$ $\mathrm{Hz}$ ), 7.38 (d, 2H, $J=8.4 \mathrm{~Hz}), 7.45-7.51$ (m, $2 \mathrm{H}), 7.72$ (t, $1 \mathrm{H}, J=7.8 \mathrm{~Hz}), 7.91(\mathrm{~d}, 1 \mathrm{H}, J=8.0 \mathrm{~Hz}) .{ }^{13} \mathrm{C} \mathrm{NMR}$ (100 MHz, DMSO-d $\mathrm{d}_{6}$ ): $\delta$ (ppm) 57.94, 103.94, 113.39, 117.06, 119.54, 123.01, 125.18, 128.92, 130.12, 132.18, 133.51, 142.80, 152.64, 154.02, 158.34, 158.38, 160.03.

2-Amino-4-(3-chlorophenyl)-4,5-dihydro-5-oxopyra no[3,2-c]chromene-3-carbonitrile 1c: $\mathrm{mp} 241^{\circ} \mathrm{C}-243^{\circ} \mathrm{C}$, (Lit.: $246^{\circ} \mathrm{C}-248^{\circ} \mathrm{C}$, [26]). ${ }^{1} \mathrm{H}$ NMR (400 MHz, DMSO$\left.\mathrm{d}_{6}\right): \delta(\mathrm{ppm}) 4.52(\mathrm{~s}, 1 \mathrm{H}), 7.26(\mathrm{~d}, 1 \mathrm{H}, J=7.2 \mathrm{~Hz}), 7.31$ - 7.38 (m, 3 H), 7.47 - 7.53 (m, 2 H), 7.73 (t, 1H, $J=7.6$ $\mathrm{Hz}), 7.91$ (d, $1 \mathrm{H}, J=7.6 \mathrm{~Hz}) .{ }^{13} \mathrm{C} \mathrm{NMR}(100 \mathrm{MHz}$, DMSO- $\left.\mathrm{d}_{6}\right): \delta$ (ppm) 57.81, 103.63, 113.43, 117.02, 119.55, 123.06, 125.13, 127.03, 127.66, 128.06, 130.87, 133.48, 133.54, 146.26, 152.66, 154.19, 158.37, 158.42, 160.06.

2-Amino-4-(4-bromophenyl)-4,5-dihydro-5-oxopyra no[3,2-c]chromene-3-carbonitrile (1d): mp $254^{\circ} \mathrm{C}$ $256^{\circ} \mathrm{C}$, (Lit.: $247^{\circ} \mathrm{C}-249^{\circ} \mathrm{C}$, [26]). ${ }^{1} \mathrm{H}$ NMR (400 MHz, 
DMSO-d $\left.\mathrm{d}_{6}\right): \delta$ (ppm) 4.48 (s, $\left.1 \mathrm{H}\right), 7.25$ (d, $2 \mathrm{H}, J=8.0$ $\mathrm{Hz}), 7.46-7.52$ (m, $4 \mathrm{H}), 7.73$ (t, $1 \mathrm{H}, J=7.4 \mathrm{~Hz}), 7.91$ (d, $1 \mathrm{H}, J=7.6 \mathrm{~Hz}) \cdot{ }^{13} \mathrm{C}$ NMR (100 MHz, DMSO-d 6 ): $\delta$ (ppm) 57.85, 103.88, 113.39, 117.08, 119.54, 120.71, 123.02, 125.20, 130.50, 131.85, 133.54, 143.23, 152.65, 154.03, 158.32, 160.04 .

2-Amino-4-(2,4-dichloro-phenyl)-4,5-dihydro-5-oxo pyrano[3,2-c]chromene-3-carbonitrile (1e): $\mathrm{mp} 258^{\circ} \mathrm{C}$ - $259^{\circ} \mathrm{C}$, (Lit.: $253^{\circ} \mathrm{C}-255^{\circ} \mathrm{C}$, [30]). ${ }^{1} \mathrm{H}$ NMR (400 MHz, DMSO-d $\left.{ }_{6}\right): \delta$ (ppm) 4.98 (s, $\left.1 \mathrm{H}\right), 7.35$ - 7.41 (m, $\left.2 \mathrm{H}\right)$, $7.48-7.54$ (m, $4 \mathrm{H}), 7.60$ (d, $1 \mathrm{H}, J=2.0 \mathrm{~Hz}), 7.74$ (dt, $\left.1 \mathrm{H}, J_{l}=8.0 \mathrm{~Hz}, J_{2}=1.6 \mathrm{~Hz}\right), 7.92\left(\mathrm{dd}, 1 \mathrm{H}, J_{l}=8.0 \mathrm{~Hz}\right.$, $\left.J_{2}=1.6 \mathrm{~Hz}\right) .{ }^{13} \mathrm{C}$ NMR (100 MHz, DMSO-d 6 ): $\delta(\mathrm{ppm})$ 34.33, 56.43, 102.96, 113.29, 117.12, 119.18, 123.04, 125.25, 128.36, 129.34, 132.56, 132.88, 133.61, 133.82, 139.91, 152.70, 154.62, 158.57, 159.94 .

2-Amino-4-(3-nitrophenyl)-4,5-dihydro-5-oxopyran o[3,2-c]chromene-3-carbonitrile (1f): $\mathrm{mp} 266^{\circ} \mathrm{C}$ $267^{\circ} \mathrm{C}$, (Lit.: $262^{\circ} \mathrm{C}-264^{\circ} \mathrm{C}$, [24]). ${ }^{1} \mathrm{H}$ NMR (400 MHz, DMSO-d $\left.{ }_{6}\right): \delta$ (ppm) 4.74 (s, $\left.1 \mathrm{H}\right), 7.46$ - 7.53 (m, $\left.2 \mathrm{H}\right)$, 7.58 (s, 2H, NH$\left.)_{2}\right), 7.64$ (t, $1 \mathrm{H}, J=8.0 \mathrm{~Hz}$ ), 7.74 (dt, $1 \mathrm{H}$, $\left.J_{l}=7.9 \mathrm{~Hz}, J_{2}=1.4 \mathrm{~Hz}\right), 7.82(\mathrm{~d}, 1 \mathrm{H}, J=7.6 \mathrm{~Hz}), 7.93$ (dd, $1 \mathrm{H}, J_{l}=8.0 \mathrm{~Hz}, J_{2}=1.2 \mathrm{~Hz}$ ), $8.12-8.15$ (m, $2 \mathrm{H}$ ). ${ }^{13} \mathrm{C}$ NMR (100 MHz, DMSO-d $\left.{ }_{6}\right): \delta$ (ppm) 57.36, 103.33, $113.39,117.07,119.45,122.77,122.93,123.10,125.19$, 130.56, 133.61, 135.26, 145.96, 148.30, 152.73, 154.35, 158.52, 158.57, 160.10.

2-Amino-4-(4-nitrophenyl)-4,5-dihydro-5-oxopyran o[3,2-c]chromene-3-carbonitrile (1g): mp $259^{\circ} \mathrm{C}$ $261^{\circ} \mathrm{C}$, (Lit.: $258^{\circ} \mathrm{C}-260^{\circ} \mathrm{C}$, [24]). ${ }^{1} \mathrm{H}$ NMR (400 MHz, DMSO-d $\left.{ }_{6}\right): \delta$ (ppm) 4.68 (s, $\left.1 \mathrm{H}\right), 7.47$ - 7.54 (m, $\left.2 \mathrm{H}\right)$, 7.58 - 7.61 (m, $2 \mathrm{H}), 7.75$ (dt, $1 \mathrm{H}, J_{1}=8.0 \mathrm{~Hz}, J_{2}=1.2$ $\mathrm{Hz}$ ), 7.93 (dd, $1 \mathrm{H}, J_{l}=8.0 \mathrm{~Hz}, J_{2}=1.2 \mathrm{~Hz}$ ), 8.21 (d, $2 \mathrm{H}$, $J=8.4 \mathrm{~Hz}) .{ }^{13} \mathrm{C}$ NMR (100 MHz, DMSO- $\left.\mathrm{d}_{6}\right): \delta(\mathrm{ppm})$ 57.20, 103.27, 113.36, 117.13, 119.34, 123.09, 124.22, $125.26,129.67,133.69,147.08,151.23,152.74,154.42$, 158.43, 158.47, 160.07.

2-Amino-4-(2-nitrophenyl)-4,5-dihydro-5-oxopyran o[3,2-c]chromene-3-carbonitrile (1h): mp $258^{\circ} \mathrm{C}$ $260^{\circ} \mathrm{C}$, (Lit.: $258^{\circ} \mathrm{C}-260^{\circ} \mathrm{C}$, [31]). ${ }^{1} \mathrm{H}$ NMR (400 MHz, DMSO-d $\left.{ }_{6}\right): \delta$ (ppm) 5.25 (s, $\left.1 \mathrm{H}\right), 7.46-7.59(\mathrm{~m}, 6 \mathrm{H})$, 7.67 (t, $1 \mathrm{H}, J=7.6 \mathrm{~Hz}), 7.73$ (t, $1 \mathrm{H}, J=7.8 \mathrm{~Hz}), 7.91$ (d, $2 \mathrm{H}, J=7.6 \mathrm{~Hz}) \cdot{ }^{13} \mathrm{C}$ NMR (100 MHz, DMSO-d 6 ): $\delta$ (ppm) 56.52, 103.75, 113.27, 117.09, 119.22, 123.03, 124.45, 125.21, 128.93, 131.65, 133.57, 134.16, 137.83, 149.66, 152.63, 154.06, 159.08, 160.19.

2-Amino-4-p-tolyl-4,5-dihydro-5-oxopyrano[3,2-c]c hromene-3-carbonitrile (1i): $\mathrm{mp} 253^{\circ} \mathrm{C}-255^{\circ} \mathrm{C}$, (Lit.: $259^{\circ} \mathrm{C}-260^{\circ} \mathrm{C}$, [28]). ${ }^{1} \mathrm{H}$ NMR (400 MHz, DMSO-d $\left.\mathrm{d}_{6}\right): \delta$ (ppm) 2.27 (s, 3H), 4.41 (s, $1 \mathrm{H}), 7.11$ - 7.15 (m, $4 \mathrm{H})$, 7.40 (s, $2 \mathrm{H}, \mathrm{NH}_{2}$ ), 7.46-7.52 (m, $\left.2 \mathrm{H}\right), 7.72$ (dt, $1 \mathrm{H}, J_{1}=$ $7.8 \mathrm{~Hz}, J_{2}=1.6 \mathrm{~Hz}$ ), $7.91\left(\mathrm{dd}, 1 \mathrm{H}, J_{I}=7.8 \mathrm{~Hz}, J_{2}=1.4\right.$ $\mathrm{Hz}) .{ }^{13} \mathrm{C}$ NMR (100 MHz, DMSO-d $\mathrm{d}_{6}$ ): $\delta$ (ppm) 21.10, 58.53, 104.59, 113.42, 117.03, 119.71, 122.94, 125.15,
128.00, 129.55, 133.38, 136.78, 140.86, 152.56, 153.72, $158.30,160.01$.

2-Amino-4-(3,4,5-trimethoxy-phenyl)-4,5-dihydro-5 -oxopyrano[3,2-c]chromene-3-carbonitrile (1j): $\mathrm{mp}$ $236^{\circ} \mathrm{C}-238^{\circ} \mathrm{C}$, (Lit.: $236^{\circ} \mathrm{C}-238^{\circ} \mathrm{C}$, [31]). ${ }^{1} \mathrm{H}$ NMR (400 MHz, DMSO-d $\left.\mathrm{d}_{6}\right): \delta$ (ppm) 3.64 (s, $\left.3 \mathrm{H}\right), 3.72$ (s, 6 $\mathrm{H}), 4.44$ (s, $1 \mathrm{H}), 6.53$ (s, $2 \mathrm{H}), 7.41$ (s, $2 \mathrm{H}, \mathrm{NH}_{2}$ ), 7.47-7.52 (m, $2 \mathrm{H}), 7.73\left(\mathrm{dt}, 1 \mathrm{H}, J_{l}=8.0 \mathrm{~Hz}, J_{2}=1.6\right.$ $\mathrm{Hz}), 7.91$ (dd, $\left.1 \mathrm{H}, J_{1}=8.0 \mathrm{~Hz}, J_{2}=1.6 \mathrm{~Hz}\right) .{ }^{13} \mathrm{C} \mathrm{NMR}$ (100 MHz, DMSO-d $\left.\mathrm{d}_{6}\right): \delta(\mathrm{ppm})$ 56.36, 58.32, 60.39, 104.11, 105.38, 113.54, 117.05, 119.71, 123.04, 125.11, 133.39, 137.03, 139.46, 152.64, 153.30, 153.98, 158.38, 160.14 .

2-Amino-4-(4-hydroxy-phenyl)-4,5-dihydro-5-oxop yrano[3,2-c]chromene-3-carbonitrile (1k): mp $266^{\circ} \mathrm{C}$ $268^{\circ} \mathrm{C}$, (Lit.: $260^{\circ} \mathrm{C}-263^{\circ} \mathrm{C}$, [28]). ${ }^{1} \mathrm{H}$ NMR (400 MHz, DMSO-d $\left.{ }_{6}\right): \delta$ (ppm) 4.33 (s, $\left.1 \mathrm{H}\right), 6.71$ (d, $2 \mathrm{H}, J=8.4$ $\mathrm{Hz}), 7.06$ (d, $0.72 \mathrm{H}, J=8.8 \mathrm{~Hz}), 7.36$ (s, $2 \mathrm{H}, \mathrm{NH}_{2}$ ), $7.43-7.49(\mathrm{~m}, 2 \mathrm{H}), 7.69\left(\mathrm{dt}, 1 \mathrm{H}, J_{1}=7.8 \mathrm{~Hz}, J_{2}=1.6\right.$ $\mathrm{Hz}), 7.89$ (dd, $1 \mathrm{H}, J_{l}=7.8 \mathrm{~Hz}, J_{2}=1.4 \mathrm{~Hz}$ ), $9.41(\mathrm{~s}, 1 \mathrm{H}$, OH). ${ }^{13} \mathrm{C}$ NMR (100 MHz, DMSO-d $\left.{ }_{6}\right): \delta$ (ppm) 58.84, $104.92,113.43,115.59,116.96,119.87,122.89,125.09$, $129.20,133.25,134.23,152.48,153.42,156.80,158.32$, 160.02 .

\subsection{General Procedure for the Synthesis of Dihydropyrano[2,3-c]pyrazoles}

To a mixture of aromatic aldehyde (1 mmol), malonitrile (1 mmol), and 3-methyl-1-phenyl-1H-pyrazol-5(4 H)-one (1 mmol), catalyst [Sipim] $\mathrm{HSO}_{4}(0.15 \mathrm{~g}, 0.12 \mathrm{mmol}$ of $\mathrm{H}^{+}$) was added and the mixture was heated at $110^{\circ} \mathrm{C}$ under solvent-free conditions. After completion of the reaction, as indicated by TLC, ethanol $(10 \mathrm{~mL})$ was added and the reaction mixture was filtered. The remaining was washed with warm ethanol $(3 \times 5 \mathrm{~mL})$ in order to separate heterogeneous catalyst. After cooling the crude products were precipitated. The crude products were purified by recrystallization from ethanol (95\%). The recovered catalyst was dried and reused for subsequent runs.

6-Amino-3-methyl-1,4-diphenyl-1,4-dihydro-pyran o[2,3-c]pyrazol-5-carbonitrile (5a): $\mathrm{mp} 170^{\circ} \mathrm{C}-171^{\circ} \mathrm{C}$, (Lit.: $168^{\circ} \mathrm{C}-170^{\circ} \mathrm{C}$, [38]). ${ }^{1} \mathrm{H}$ NMR (400 $\left.\mathrm{MHz} \mathrm{CDCl}_{3}\right)$ : $\delta(\mathrm{ppm}) 1.92$ (s, $3 \mathrm{H}), 4.69(\mathrm{~s}, 1 \mathrm{H}), 4.71\left(\mathrm{~s}, 2 \mathrm{H}, \mathrm{NH}_{2}\right)$, $7.27-7.40$ (m, $6 \mathrm{H}), 7.49$ (t, $2 \mathrm{H}, J=7.8 \mathrm{~Hz}), 7.68$ (d, $2 \mathrm{H}, J=8.0 \mathrm{~Hz}) .{ }^{13} \mathrm{C} \mathrm{NMR}\left(100 \mathrm{MHz}, \mathrm{CDCl}_{3}\right): \delta(\mathrm{ppm})$ 12.94, 37.44, 63.88, 98.36, 119.14, 121.22, 126.80, 127.62, 127.91, 128.83, 129.31, 137.57, 142.00, 143.84, 146.46, 158.17.

6-Amino-4-(4-chlorophenyl)-3-methyl-1-phenyl-1,4dihydro-pyrano[2,3-c]pyrazol-5-carbonitrile (5b): $\mathrm{mp}$ $174^{\circ} \mathrm{C}-176^{\circ} \mathrm{C}$, (Lit.: $174^{\circ} \mathrm{C}-177^{\circ} \mathrm{C}$, [43]). ${ }^{1} \mathrm{H}$ NMR (400 MHz, $\left.\mathrm{CDCl}_{3}\right): \delta(\mathrm{ppm}) 1.92$ (s, $\left.3 \mathrm{H}\right), 4.68$ (s, $\left.1 \mathrm{H}\right)$, 4.74 (s, $2 \mathrm{H}, \mathrm{NH}_{2}$ ), 7.22 (d, 2H, $J=8.4 \mathrm{~Hz}$ ), 7.33-7.37 (m, 
3H), 7.49 (t, $2 \mathrm{H}, J=7.8 \mathrm{~Hz}$ ), 7.67 (d, $2 \mathrm{H}, J=8.0 \mathrm{~Hz}$ ). ${ }^{13} \mathrm{C}$ NMR (100 MHz, $\mathrm{CDCl}_{3}$ ): $\delta$ (ppm) 12.97, 36.91, 63.36, 97.87, 118.95, 121.25, 126.92, 129.06, 129.28, 129.34, 133.44, 137.45, 140.57, 143.80, 146.28, 158.23.

6-Amino-4-(2,4-dichlorophenyl)-3-methyl-1-phenyl1,4-dihydro-pyrano[2,3-c]pyrazol-5-carbonitrile (5c): mp $183^{\circ} \mathrm{C}-185^{\circ} \mathrm{C}$, (Lit.: $182^{\circ} \mathrm{C}-184^{\circ} \mathrm{C}$, [38]). ${ }^{1} \mathrm{H}$ NMR $\left(400 \mathrm{MHz}, \mathrm{CDCl}_{3}\right): \delta(\mathrm{ppm}) 1.92(\mathrm{~s}, 3 \mathrm{H}), 4.78(\mathrm{~s}, 2 \mathrm{H}$, $\mathrm{NH}_{2}$ ), 5.29 (s, 1H), 7.17 (d, $1 \mathrm{H}, J=8.4 \mathrm{~Hz}$ ), $7.26-7.28$ (m, $1 \mathrm{H}), 7.35$ (t, $1 \mathrm{H}, J=7.2 \mathrm{~Hz}), 7.46-7.51(\mathrm{~m}, 3 \mathrm{H})$, 7.67 (d, $2 \mathrm{H}, J=8.0 \mathrm{~Hz}) .{ }^{13} \mathrm{C}$ NMR $\left(100 \mathrm{MHz}, \mathrm{CDCl}_{3}\right): \delta$ (ppm) 12.77, 33.53, 61.98, 97.58, 118.62, 121.28, 126.97, 128.04, 129.35, 129.68, 131.49, 133.95, 137.41, 137.86, 143.95, 146.04, 158.84.

6-Amino-4-(4-bromophenyl)-3-methyl-1-phenyl-1,4dihydro-pyrano[2,3-c]pyrazol-5-carbonitrile (5d): $\mathrm{mp}$ $182^{\circ} \mathrm{C}-184^{\circ} \mathrm{C}$, (Lit.: $176^{\circ} \mathrm{C}-177^{\circ} \mathrm{C}$, [40]). ${ }^{1} \mathrm{H}$ NMR $\left(400 \mathrm{MHz}, \mathrm{CDCl}_{3}\right): \delta(\mathrm{ppm}) 1.92(\mathrm{~s}, 3 \mathrm{H}), 4.67(\mathrm{~s}, 1 \mathrm{H})$, 4.73 (s, 2H, NH$\left.)_{2}\right), 7.17$ (d, 2H, $J=8.0 \mathrm{~Hz}$ ), $7.35(\mathrm{t}, 1 \mathrm{H}$, $J=7.4 \mathrm{~Hz}), 7.48-7.53$ (m, 4H), 7.67 (d, $2 \mathrm{H}, J=7.6 \mathrm{~Hz})$. ${ }^{13} \mathrm{C}$ NMR (100 MHz, $\left.\mathrm{CDCl}_{3}\right): \delta$ (ppm) 12.98, 36.98, 63.31, 97.78, 118.92, 121.26, 121.60, 126.93, 129.34, 129.63, 132.01, 137.45, 141.08, 146.28, 158.22.

6-Amino-4-(4-fluorophenyl)-3-methyl-1-phenyl-1,4dihydro-pyrano[2,3-c]pyrazol-5-carbonitrile (5e): $\mathrm{mp}$ $170^{\circ} \mathrm{C}-171^{\circ} \mathrm{C}$, (Lit.: $167^{\circ} \mathrm{C}-168^{\circ} \mathrm{C}$, [38]). ${ }^{1} \mathrm{H}$ NMR $\left(400 \mathrm{MHz}, \mathrm{CDCl}_{3}\right): \delta$ (ppm) 1.91 (s, 3H), $4.69(\mathrm{~s}, 1 \mathrm{H})$, $4.73\left(\mathrm{~s}, 2 \mathrm{H}, \mathrm{NH}_{2}\right.$ ), 7.07 (t, $2 \mathrm{H}, J=8.6 \mathrm{~Hz}$ ), $7.24-7.28$ (m, $2 \mathrm{H}), 7.35$ (t, $1 \mathrm{H}, J=7.4 \mathrm{~Hz}), 7.49$ (t, 2H, $J=7.8$ $\mathrm{Hz}), 7.67$ (d, $2 \mathrm{H}, J=8.0 \mathrm{~Hz}) \cdot{ }^{13} \mathrm{C}$ NMR $(100 \mathrm{MHz}$, $\left.\mathrm{CDCl}_{3}\right): \delta(\mathrm{ppm}) 12.94,36.78,63.69,98.15,115.86$, 119.01, 121.24, 126.88, 129.33, 129.54, 137.49, 143.77, 146.32, 158.14, 162.17 (d, $\left.J_{\mathrm{C}-\mathrm{F}}=244.0 \mathrm{~Hz}\right)$.

6-Amino-3-methyl-4-(3-nitrophenyl)-1-phenyl-1,4-d ihydro-pyrano[2,3-c]pyrazol-5-carbonitrile (5f): $\mathrm{mp}$ $190^{\circ} \mathrm{C}-191^{\circ} \mathrm{C}$, (Lit.: $190^{\circ} \mathrm{C}-192^{\circ} \mathrm{C}$, [43]). ${ }^{1} \mathrm{H}$ NMR $\left(400 \mathrm{MHz}, \mathrm{CDCl}_{3}\right): \delta$ (ppm) 1.92 (s, $\left.3 \mathrm{H}\right), 4.84$ (s, 3H, $\mathrm{CH} \& \mathrm{NH}_{2}$ ), 7.37 (t, $\left.1 \mathrm{H}, J=7.4 \mathrm{~Hz}\right), 7.51(\mathrm{t}, 2 \mathrm{H}, J=$ $7.6 \mathrm{~Hz}$ ), 7.60 (t, $1 \mathrm{H}, J=8.0 \mathrm{~Hz}), 7.67-7.70(\mathrm{~m}, 3 \mathrm{H})$, 8.14 (s, $1 \mathrm{H}), 8.21$ (d, $1 \mathrm{H}, J=8.0 \mathrm{~Hz}) .{ }^{13} \mathrm{C}$ NMR $(100$ $\mathrm{MHz}, \mathrm{CDCl}_{3}$ ): $\delta$ (ppm) 13.01, 37.38, 62.11, 97.21, 118.76, 121.42, 122.87, 127.11, 129.37, 129.89, 134.17, 137.33, 144.49, 145.92, 148.76, 158.75.

6-Amino-3-methyl-4-(4-nitrophenyl)-1-phenyl-1,4-d ihydro-pyrano[2,3-c]pyrazol-5-carbonitrile (5g): $\mathrm{mp}$ $194^{\circ} \mathrm{C}-196^{\circ} \mathrm{C}$, (Lit.: $195^{\circ} \mathrm{C}-197^{\circ} \mathrm{C}$, [43]). ${ }^{1} \mathrm{H}$ NMR (400 MHz, $\left.\mathrm{CDCl}_{3}\right): \delta(\mathrm{ppm}) 1.92(\mathrm{~s}, 3 \mathrm{H}), 4.83(\mathrm{~s}, 3 \mathrm{H}$, $\mathrm{CH} \& \mathrm{NH}_{2}$ ), 7.37 (t, $1 \mathrm{H}, J=7.4 \mathrm{~Hz}$ ), $7.47-7.53$ (m, 4 H), 7.78 (d, $2 \mathrm{H}, J=8.0 \mathrm{~Hz}), 8.27$ (d, $2 \mathrm{H}, J=8.4 \mathrm{~Hz}$ ). ${ }^{13} \mathrm{C}$ NMR (100 MHz, $\mathrm{CDCl}_{3}$ ): $\delta$ (ppm) 12.98, 37.33, 62.26, 97.07, 118.58, 121.35, 124.28, 127.16, 128.88, 129.40, 137.30, 143.83, 146.00, 147.46, 149.19, 158.56 .

6-Amino-4-(3,4-dimethoxyphenyl)-3-methyl-1-phen yl-1,4-dihydro-pyrano[2,3-c]pyrazol-5-carbonitrile (5h): $\mathrm{mp} 167^{\circ} \mathrm{C}-169^{\circ} \mathrm{C}$, (Lit.: $193^{\circ} \mathrm{C}-195^{\circ} \mathrm{C}$, [38]). ${ }^{1} \mathrm{H}$ NMR (400 MHz, $\mathrm{CDCl}_{3}$ ): $\delta$ (ppm) 1.91 (s, $3 \mathrm{H}$ ), 3.85 (s, $3 \mathrm{H}), 3.87$ (s, $3 \mathrm{H}$ ), 4.62 (s, $1 \mathrm{H}), 4.73$ (s, $\left.2 \mathrm{H}, \mathrm{NH}_{2}\right), 6,73$ (d, $1 \mathrm{H}, J=1.6 \mathrm{~Hz}), 6.79-6.85$ (m, 2H), 7.31 (t, $1 \mathrm{H}, J=$ $8.0 \mathrm{~Hz}), 7.45$ (t, $2 \mathrm{H}, J=8.0 \mathrm{~Hz}), 7.66(\mathrm{~d}, 2 \mathrm{H}, J=8.4$ $\mathrm{Hz}) .{ }^{13} \mathrm{C}$ NMR (100 MHz, $\left.\mathrm{CDCl}_{3}\right): \delta(\mathrm{ppm}) 12.98,37.06$, 55.86, 55.98, 63.92, 98.29, 110.82, 111.12, 119.22, $120.18,121.05,126.71,129.28,134.56,137.57,143.80$, 146.52, 148.41, 149.20, 158.02 .

6-Amino-4-(4-hydroxyphenyl)-3-methyl-1-phenyl-1, 4-dihydro-pyrano[2,3-c]pyrazol-5-carbonitrile (5i): mp $206^{\circ} \mathrm{C}-208^{\circ} \mathrm{C}$, (Lit.: $206^{\circ} \mathrm{C}-207^{\circ} \mathrm{C}$, [40]). ${ }^{1} \mathrm{H}$ NMR (500 MHz, DMSO-d 6 ): $\delta$ (ppm) 1.78 (s, $3 \mathrm{H}), 4.55$ (s, 1 H), 6.71 (d, $2 \mathrm{H}, J=7.8 \mathrm{~Hz}), 7.03$ (d, $2 \mathrm{H}, J=7.8 \mathrm{~Hz}$ ), $7.10\left(\mathrm{~s}, 2 \mathrm{H}, \mathrm{NH}_{2}\right), 7.31$ (t, $\left.1 \mathrm{H}, J=7.0 \mathrm{~Hz}\right), 7.48(\mathrm{t}, 2 \mathrm{H}$, $J=7.3 \mathrm{~Hz}), 7.77(\mathrm{~d}, 2 \mathrm{H}, J=7.7 \mathrm{~Hz}), 9.31(\mathrm{~s}, 1 \mathrm{H}) .{ }^{13} \mathrm{C}$ NMR (125 MHz, DMSO-d $\mathrm{d}_{6}$ ): $\delta$ (ppm) 13.45, 36.88, 59.68, 99.91, 116.07, 120.75, 120.96, 126.94, 129.62, 130.18, 134.79, 138.45, 144.65, 146.22, 157.15, 160.305.

\section{Acknowledgements}

We are thankful to Persian Gulf University Research Council for partial support of this work.

\section{REFERENCES}

[1] P. Wasserscheid and T. Welton, "Ionic Liquids in Synthesis,” 2nd Edition, Wiley-VCH, Weinheim, 2007. doi:10.1002/9783527621194

[2] W. Keim, W. Korth and P. Wasserscheid, "Ionic Liquids," Patent WO/2000/016902, March 30, 2000.

[3] P. Wasserscheid, M. Sesing and W. Korth, "Hydrogensulfate and Tetrakis(Hydrogensulfato)borate Ionic Liquids: Synthesis and Catalytic Application in Highly BrønstedAcidic Systems for Friedel-Crafts Alkylation," Green Chemistry, Vol. 4, No. 2, 2002, pp. 134-138. doi:10.1039/b109845b

[4] N. Gupta, G. L. Sonu, J. Kad and Singh, “Acidic Ionic Liquid [bmim] $\mathrm{HSO}_{4}$ : An Efficient Catalyst for Acetalization and Thioacetalization of Carbonyl Compounds and Their Subsequent Deprotection," Catalysis Communications, Vol. 8, No. 9, 2007, pp. 1323-1328. doi:10.1016/j.catcom.2006.11.030

[5] D. Q. Xu, W. L. Yang, S. P. Luo, B. T. Wang, M. Wu and Z. Y. Xu, "Fischer Indole Synthesis in Brønsted Acidic Ionic Liquids: A Green, Mild, and Regiospecific Reaction System,” European Journal of Organic Chemistry, No. 6, 2007, pp. 1007-1012. doi:10.1002/ejoc.200600886

[6] W. Wang, W. Cheng, L. Shao and J. Yang, “[TMBSA] $\left[\mathrm{HSO}_{4}\right]$ Ionic Liquid as Novel Catalyst for the Rapid Acetylation of Alcohols, Hydroxyesters and Phenols under Solvent-Free Conditions," Catalysis Letters, Vol. 121, No. 1-2, 2008, pp. 77-80. doi:10.1007/s10562-007-9295-2

[7] A. R. Hajipour, A. Rajaei and A. E. Ruoho, “A Mild and Efficient Method for Preparation of Azides from Alcohols 
Using Acidic Ionic Liquid [H-NMP]HSO ${ }_{4}$, "Tetrahedron Letters, Vol. 50, No. 6, 2009, pp. 708-711. doi:10.1016/j.tetlet.2008.11.111

[8] H. Tajik, K. Niknam and F. Parsa, "Using Acidic Ionic Liquid 1-Butyl-3-Methylimidazolium Hydrogen Sulfate in Selective Nitration of Phenols under Mild Conditions," Journal of the Iranian Chemical Society, Vol. 6, No. 1, 2009, pp. 159-164.

[9] K. Niknam and M. Damya, "1-Butyl-3-Methylimidazolium Hydrogen Sulfate [bmim] $\mathrm{HSO}_{4}$ : An Efficient Reusable Acidic Ionic Liquid for the Synthesis of 1,8-DioxoOctahydroxanthenes," Journal of the Chinese Chemical Society, Vol. 56, No. 3, 2009, pp. 659-665.

[10] K. Niknam, M. A. Zolfigol, D. Saberi and M. Khonbazi, "1-Butyl-3-Methylimidazolium Hydrogen Sulfate [bmim] HSO4: An Efficient Reusable Acidic Ionic Liquid for the Formylation of Alcohols," Chinese Journal of Chemistry, Vol. 27, No. 8, 2009, pp. 1548-1552. doi:10.1002/cjoc.200990261

[11] H. Tajik, K. Niknam and M. Sarrafan, “1-Butyl-3-Me- thylimidazolium Hydrogen Sulfate ([bmim]-HSO ${ }_{4}$ ) Mediated Synthesis of Polysubstituted Quinolines," Synthetic Communications, Vol. 41, No. 14, 2011, pp. 2103-2114. doi:10.1080/00397911.2010.497596

[12] K. Qiao, H. Hagiwara and C. Yokoyama, “Acidic Ionic Liquid Modified Silica Gel as Novel Solid Catalysts for Esterification and Nitration Reactions," Journal of Molecular Catalyst A: Chemicals, Vol. 246, No. 1-2, 2006, pp. 65-69. doi:10.1016/j.molcata.2005.07.031

[13] R. Sugimura, K. Qiao, D. Tomida and C. Yokoyama, "Immobilization of Acidic Ionic Liquids by Copolymerization with Styrene and Their Catalytic Use for Acetal formation," Catalysis Communications, Vol. 8, No. 5, 2007, pp. 770-772. doi:10.1016/j.catcom.2006.08.049

[14] A. Chrobok, S. Baj, W. Pudlo and A. Jarzebski, "Supported Hydrogensulfate Ionic Liquid Catalysis in BaeyerVilliger Reaction," Applied Catalysis A: General, Vol. 366, No. 1, 2009, pp. 22-28. doi:10.1016/j.apcata.2009.06.040

[15] M. N. Sefat, D. Saberi and K. Niknam, "Preparation of Silica-Based Ionic Liquid an Efficient and Recyclable Catalyst for One-Pot Synthesis of a-Aminonitriles," Catalysis Letters, Vol. 141, No. 11, 2011, pp. 1713-1720. doi:10.1007/s10562-011-0696-X

[16] M. Baghernejad and K. Niknam, “Synthesis of 4,4'-(Arylmethylene)bis(1H-pyrazol-5-ols) Using Silica-Bonded Ionic Liquid as Recyclable Catalyst," International Journal of Chemistry, Vol. 4, No. 3, 2012, pp. 52-60. doi:10.5539/ijc.v4n3p52

[17] G. R. Green, J. M. Evans and A. K. Vong, "Comprehensive Heterocyclic Chemistry II,” Pergamon Press, Oxford, 1995, p. 469.

[18] W. O. Foye, "Principi di Chemico Farmaceutica," Piccin, Padova, 1991, p. 416.

[19] L. Bonsignore, G. Loy, D. Secci and A. Calignano, "Synthesis and Pharmacological Activity of 2-Oxo-(2H) 1benzopyran-3-Carboxamide Derivatives," European Journal of Medicinal Chemistry, Vol. 28, No. 6, 1993, pp. 517-520. doi:10.1016/0223-5234(93)90020-F
[20] C. S. Konkoy, D. B. Fick, S. X. Cai, N. C. Lan and J. F. W. Keana, PCT Int Appl, WO 0075123, 2000, [Chem Abstr, Vol. 134, 2001, 29313a].

[21] D. Arnesto, W. M. Horspool, N. Martin, A. Ramos and C. Seaone, "Synthesis of Cyclobutenes by the Novel PhotoChemical Ring Contraction of 4-Substituted 2-Amino-3,5Dicyano-6-Phenyl-4H-Pyrans," The Journal of Organic Chemistry, Vol. 54, No. 13, 1989, pp. 3069-3072. doi:10.1021/jo00274a021

[22] R. M. Shaker, "Synthesis and Reactions of Some New 4H-Pyranol[3,2-c]benzopyran-5-One Derivatives and Their Potential Biological Activities,” Pharmazie, Vol. 51, No. 3, 1996, pp. 148-151.

[23] M. Kidwai and S. Saxena, "Convenient Preparation of Pyrano Benzopyranes in Aqueous Media," Synthetic Communications, Vol. 36, No. 18, 2006, pp. 2737-2742. doi:10.1080/00397910600764774

[24] S. Abdolmohammadi and S. Balalaie, "Novel and Efficient Catalysts for the One-Pot Synthesis of 3,4-Dihydropyrano[c]chromene Derivatives in Aqueous Media," Tetrahedron Letters, Vol. 48, No. 18, 2007, pp. 32993303. doi:10.1016/j.tetlet.2007.02.135

[25] J. M. Khurana and S. Kumar, “Tetrabutylammonium Bromide (TBAB): A Neutral and Efficient Catalyst for the Synthesis of Biscoumarin and 3,4-Dihydropyrano[c]-Chromene Derivatives in Water and Solvent-Free Conditions," Tetrahedron Letters, Vol. 50, No. 28, 2009, pp. 41254127. doi:10.1016/j.tetlet.2009.04.125

[26] H. Mehrabi and H. Abusaidi, "Synthesis of Biscoumarin and 3,4-Dihydropyrano[c]chromene Derivatives Catalysed by Sodium Dodecyl Sulfate (SDS) in Neat Water," Journal of the Iranian Chemical Society, Vol. 7, No. 4, 2010, pp. 890-894.

[27] J. M. Khurana, B. Nand and P. Saluja, "DBU: A Highly Efficient Catalyst for One-Pot Synthesis of Substituted 3,4-dihydropyrano[3,2-c]chromenes, Dihydropyrano- [4,3-b] pyranes, 2-Amino-4Hbenzo[h]chromenes and 2-Amino4H benzo[g]chromenes in Aqueous Medium,” Tetrahedron, Vol. 66, No. 30, 2010, pp. 5637-5641. doi:10.1016/j.tet.2010.05.082

[28] M. M. Heravi, M. Zakeri and N. Mohammadi, "Morpholine Catalyzed One-pot Multicomponent Synthesis of Compounds Containing Chromene Core in Water," Chinese Journal of Chemistry, Vol. 29, No. 6, 2011, pp. 1163-1166. doi:10.1002/cjoc.201190217

[29] H. Nagabhushana, S. S. Saundalkar, L. Muralidhar, B. M. Nagabhushana, C. R. Girija, D. Nagaraja, M. A. Pasha and V. P. Jayashankara, "a- $-\mathrm{Fe}_{2} \mathrm{O}_{3}$ Nanoparticles: An Efficient, Inexpensive Catalyst for the One-Pot Preparation of 3,4-Dihydropyrano[c]chromenes," Chinese Chemical Letters, Vol. 22, No. 2, 2011, pp. 143-146. doi:10.1016/j.cclet.2010.09.020

[30] H. Mehrabi and M. Kazemi-Mireki, "CuO Nanoparticles: An Efficient and Recyclable Nanocatalyst for the Rapid and Green Synthesis of 3,4-Dihydropyrano[c]chromenes," Chinese Chemical Letters, Vol. 22, No. 12, 2011, pp. 1419-1422. doi:10.1016/j.cclet.2011.06.003

[31] K. Niknam and A. Jamali, "Silica-Bonded $N$-Propylpiperazine Sodium n-Propionate as Recyclable Basic Catalyst 
for Synthesis of 3,4-Dihydropyrano[c]chromene Derivatives and Biscoumarins,“ Chinese Journal of Catalysis, Vol. 33, No. 11, 2012, pp. 1840-1849. doi:10.1016/S1872-2067(11)60457-9

[32] M. H. Elnagdi, M. R. H. Elmaoghayer and G. E. H. Elgemeie, "Chemistry of Pyrazolopyrimidines," Advance in Heterocyclic Chemistry, Vol. 41, 1987, pp. 319-376. doi:10.1016/S0065-2725(08)60164-6

[33] F. Karci and F. Karci, "Synthesis of Some Novel Pyrazolo[5,1-c][1,2,4]triazine Derivatives and Investigation of Their Absorption Spectra," Dyes and Pigments, Vol. 76, No. 1, 2008, pp. 97-103. doi:10.1016/j.dyepig.2006.08.011

[34] S. G. Kuo, L. J. Huang and H. Nakamura, "Studies on Heterocyclic Compounds. 6. Synthesis and Analgesic and Antiinflammatory Activities of 3,4-Dimethylpyrano- [2,3-c] pyrazol-6-one Derivatives," Journal of Medicinal Chemistry, Vol. 27, No. 4, 1984, pp. 539-544. doi:10.1021/jm00370a020

[35] A. A. Fadda, A. A. H. Abdel-Rahman, E. A. Hamed and E. H. Khalil, "Utility of Enaminonitriles in Heterocyclic Synthesis: Synthesis and Antimicrobial Activity of Some New Azole and Azine Derivatives,” American Journal of Organic Chemistry, Vol. 2, No. 2, 2012, pp. 7-13. doi:10.5923/j.ajoc.20120202.02

[36] N. M. Abunada, H. M. Hassaneen, N. G. Kandile and O. A. Miqdad, "Synthesis and Antimicrobial Activity of Some New Pyrazole, Fused Pyrazolo[3,4-d]-pyrimidine and Pyrazolo[4,3-e][1,2,4]-triazolo[1,5-c]pyrimidine Derivatives,” Molecules, Vol. 13, No. 7, 2008, pp. 15011517. doi:10.3390/molecules13071501

[37] N. J. Thumar and M. P. Patel, "Synthesis and in Vitro Antimicrobial Evaluation of 4H-Pyrazolopyran, Benzopyran and Naphthopyran Derivatives of 1H-Pyrazole," Arkivoc, No. 13, 2009, pp. 363-380.

[38] D. Shi, J. Mou, Q. Zhuang, L. Niu, N. Wu and X. Wang, "Three-Component One-Pot Synthesis of 1,4-Dihydropyrano[2,3-c]pyrazole Derivatives in Aqueous Media," Synthetic Communications, Vol. 34, No. 24, 2004, pp. 4557-4563. doi:10.1081/SCC-200043224

[39] T. S. Jin, A. Q. Wang, Z. L. Cheng, J. S. Zhang and T. S. $\mathrm{Li}$, "A Clean and Simple Synthesis of 6-Amino-4-Aryl5-Cyano-3-Methyl-1-Phenyl-1,4-Dihydropyrano[2,3-c]Py razole in Water,” Synthetic Communications, Vol. 35, No. 1, 2005, pp. 137-143. doi:10.1081/SCC-200046527

[40] S. B. Guo, S. X. Wang and J. T. Li, "D,L-Proline-Catalyzed One-Pot Synthesis of Pyrans and Pyrano[2,3c]pyrazole Derivatives by a Grinding Method under Solvent-Free Conditions," Synthetic Communications, Vol. 37, No. 13, 2007, pp. 2111-2120.

\section{doi:10.1080/00397910701396906}

[41] F. Lehmann, M. Holm and S. Laufer, “Three-Component Combinatorial Synthesis of Novel Dihydropyrano[2,3-c] pyrazoles," Journal of Combinatorial Chemistry, Vol. 10, No. 3, 2008, pp. 364-367. doi:10.1021/cc800028m

[42] H. M. Al-Matar, K. D. Khalil, A. Y. Adam and M. H. Elnagdi, "Green One Pot Solvent-Free Synthesis of Pyrano[2,3-c]-Pyrazoles and Pyrazolo[1,5-a]Pyrimidines," Molecules, Vol. 15, No. 9, 2010, pp. 6619-6629. doi:10.3390/molecules15096619

[43] M. M. Heravi, A. Ghods, F. Derikvand, K. Bakhiari and F. F. Bamoharram, " $\mathrm{H}_{14}\left[\mathrm{NaP}_{5} \mathrm{~W}_{30} \mathrm{O}_{110}\right]$ Catalyzed One-Pot Three-Component Synthesis of Dihydropyrano[2,3-c]pyrazole and pyrano[2,3-d]pyrimidine Derivatives," Journal of the Iranian Chemical Society, Vol. 7, No. 3, 2010, pp. 615-620.

[44] A. Siddekha, A. Nizam and M. A. Pasha, “An Efficient and Simple Approach for the Synthesis of Pyranopyrazoles Using Imidazole (Catalytic) in Aqueous Medium, and the Vibrational Spectroscopic Studies on 6-Amino4-(4'Methoxyphenyl)-5-Cyano-3-Methyl-1-Phenyl-1,4-Dihydropyrano[2,3-c]pyrazole Using Density Functional Theory," Spectrochimica Acta Part A: Molecular and Biomolecular Spectroscopy, Vol. 81, No. 1, 2011, pp. 431440. doi:10.1016/j.saa.2011.06.033

[45] A. Hasaninejad, M. Shekouhy, N. Golzar, A. Zare and M. M. Doroodmand, "Silica Bonded n-Propyl-4-Aza-1-Azoniabicyclo[2.2.2]octane chloride (SB-DABCO): A Highly Efficient, Reusable and New Heterogeneous Catalyst for the Synthesis of 4H-Benzo[b]pyran Derivatives," Applied Catalysis A: General, Vol. 402, No. 1-2, 2011, pp. 11-22. doi:10.1016/j.apcata.2011.04.012

[46] H. Mecadon, M. R. Rohman, M. Rajbangshi and B. Myrboh, " $\gamma$-Alumina as a Recyclable Catalyst for the FourComponent Synthesis of 6-Amino-4-Alkyl/Aryl-3-methyl2,4-Dihydropyrano[2,3-c]pyrazole-5-carbonitriles in aqueous medium,” Tetrahedron Letters, Vol. 52, No. 19, 2011, pp. 2523-2525.

[47] S. R. Mandha, S. Siliveri, M. Alla, V. R. Bommena, M. R. Bommineni and S. Balasubramanian, "Eco-Friendly Synthesis and Biological Evaluation of Substituted Pyrano [2,3-c]pyrazoles,” Bioorganic and Medicinal Chemistry Letters, Vol. 22, No. 16, 2012, pp. 5272-5278. doi:10.1016/j.tetlet.2011.03.036

[48] S. H. S. Azzam and M. A. Pasha, "Simple and Efficient Protocol for the Synthesis of Novel Dihydro-1H-pyrano [2,3-c]pyrazol-6-Ones via a One-Pot Four-Component Reaction,” Tetrahedron Letters, Vol. 53, No. 50, 2012, pp. 6834-6837. 